\title{
Evaluation of plan robustness on the dosimetry of volumetric arc radiotherapy (VMAT) with set-up uncertainty in Nasopharyngeal carcinoma (NPC) radiotherapy
}

Zhen Ding ${ }^{*} \mathbb{0}$, Xiaoyong Xiang*, Qi Zeng, Jun Ma, Zhitao Dai, Kailian Kang and Suyan Bi

\begin{abstract}
Purpose: To evaluate the sensitivity to set up the uncertainty of VMAT plans in Nasopharyngeal carcinoma (NPC) treatment by proposing a plan robustness evaluation method.

Methods: 10 patients were selected for this study. A 2-arc volumetric-modulated arc therapy (VMAT) plan was generated for each patient using Varian Eclipse (13.6 Version) treatment planning system (TPS). 5 uncertainty plans (U-plans) were recalculated based on the first 5 times set-up errors acquired from cone-beam computer tomography (CBCT). The dose differences of the original plan and perturbed plan corresponded to the plan robustness for the structure. Tumor control probability (TCP) and normal tissues complication probability (NTCP) were calculated for biological evaluation.

Results: The mean dose differences of $D_{98 \%}$ and $D_{95 \%}\left(\Delta D_{98 \%}\right.$ and $\left.\Delta D_{95 \%}\right)$ of PTVp were respectively 3.30 Gy and 2.02 Gy. The $\Delta \mathrm{D}_{98 \%}$ and $\Delta \mathrm{D}_{95 \%}$ of CTVp were $1.12 \mathrm{~Gy}$ and $0.58 \mathrm{~Gy}$. The $\Delta \mathrm{D}_{98 \%}$ and $\Delta \mathrm{D}_{95 \%}$ of $\mathrm{CTV} \mathrm{n}$ were $1.39 \mathrm{~Gy}$ and $1.03 \mathrm{~Gy}$, distinctively lower than those in PTVn (2.8 Gy and 2.0 Gy). The CTV-to-PTV margin increased the robustness of CTVs. The $\Delta D_{98 \%}$ and $\Delta D_{95 \%}$ of GTVp were $0.56 \mathrm{~Gy}$ and $0.33 \mathrm{~Gy}$. GTVn exhibited strong robustness with little variation of $\mathrm{D}_{98 \%}(0.64 \mathrm{~Gy})$ and $\mathrm{D}_{95 \%}(0.39 \mathrm{~Gy})$. No marked mean dose variations of $\mathrm{D}_{\text {mean }}$ were seen. The mean reduction of TCP $(\triangle T C P)$ in GTVp and CTVp were respectively $0.4 \%$ and $0.3 \%$. The mean $\triangle T C P s$ of GTVn and CTVn were $0.92 \%$ and $1.3 \%$ respectively. The CTV exhibited the largest $\triangle T C P(2.2 \%)$. In OARs, the brain stem exhibited weak robustness due to their locations in the vicinity of PTV. Bilateral parotid glands were sensitive to set-up uncertainty with a mean reduction of NTCP ( $\triangle N T C P$ ) of $6.17 \%$ (left) and $7.70 \%$ (right). The $D_{\max }$ of optical nerves and lens varied slightly.

Conclusion: VMAT plans had a strong sensitivity to set-up uncertainty in NPC radiotherapy, with increasing risk of underdose of tumor and overdose of vicinal OARs. We proposed an effective method to evaluate the plan robustness of VMAT plans. Plan robustness and complexity should be taken into account in photon radiotherapy.
\end{abstract}

*Correspondence: dingzhen0909@163.com; xiangxiaoyong16@163.com

Department of Radiation Oncology, National Cancer Center/National

Clinical Research Center for Cancer/Cancer Hospital and Shenzhen

Hospital, Chinese Academy of Medical Sciences and Peking Union

Medical College, No. 113 Baohe Rd, Longgang District, Shenzhen

City 518116, Guangdong Province, People's Republic of China

(c) The Author(s) 2021. Open Access This article is licensed under a Creative Commons Attribution 4.0 International License, which permits use, sharing, adaptation, distribution and reproduction in any medium or format, as long as you give appropriate credit to the original author(s) and the source, provide a link to the Creative Commons licence, and indicate if changes were made. The images or other third party material in this article are included in the article's Creative Commons licence, unless indicated otherwise in a credit line to the material. If material is not included in the article's Creative Commons licence and your intended use is not permitted by statutory regulation or exceeds the permitted use, you will need to obtain permission directly from the copyright holder. To view a copy of this licence, visit http://creativecommons.org/licenses/by/4.0/. The Creative Commons Public Domain Dedication waiver (http://creativecommons.org/publicdomain/zero/1.0/) applies to the data made available in this article, unless otherwise stated in a credit line to the data. 
Keywords: Robustness, Tumor control probability, Normal tissue complication probability, Set-up uncertainty

\section{Introduction}

Radiotherapy (RT) is the main strategy for Nasopharyngeal carcinoma (NPC) [1]. Owing to large irradiation volumes, complex and intricate anatomical structures, precision dose coverage, and organ at risk (OAR) sparing were crucial in NPC radiotherapy [2]. Volumetric arc radiotherapy (VMAT) had been widely used in NPC radiotherapy, for VMAT performed optimized dose distribution and OAR sparing by continuous variation of multi machine parameters [3]. However, increased plan complexity elevated risk of dose calculation and delivery, for more complex plans required smaller and irregular beam apertures, larger tongue-and groove effects, and greater extent modulation of machine parameters, including gantry rotation speed, dose rate, and multi leaf collimator (MLC) position $[4,5]$. VMAT plans may show the sensitivity of dose delivery to subtle deviations, including machine parameters and target motion $[4,6]$.

Image guidance, such as the cone-beam computed tomography (CBCT) has been widely used in position verification to reduce patient set-up uncertainty [7]. The protocol of imaging frequency varies among centers to balance the treatment efficiency and accuracy. The unknown remaining fractions may result in unexpected dose deviation and potential tumor recurrence [8]. For this purpose, we aimed to study the sensitivity of highly optimized VMAT plans to geometry deviation to make a more complete description of dose delivery for complex plans.

Treatment plan robustness is the degree of resiliency of the required dose distribution to these uncertainties and varies with the treatment site, technique, and method. Yock's [9] report reviewed robustness analysis methods and their dosimetric effects, to promote reliable plan evaluation and dose reporting, particularly during clinical trials conducted across institutions and treatment modalities. The concept of robustness had been widely used in proton treatment plans for the sharp distal fall-off and scattering characteristics but was ignored in photon radiotherapy [5]. We adopted a plan robustness quantification method to address the sensitivity of VMAT plans to geometric uncertainty based on the daily CBCT shifts. Besides, the tumor control probability (TCP) and normal tissues complication probability (NTCP) models were applied to evaluate the potential biological dose differences.

\section{Methods}

\section{Patient selection and delineation}

We retrospectively evaluated treatment plans for 10 NPC patients treated in our center. The clinical characteristics of the patients enrolled in this study were shown in Table 1. All the patients were immobilized by a thermoplastic mask in a supine position. The CT image with a $2.5 \mathrm{~mm}$ slice thickness was acquired using a 16-slice CT scanner (GE Discovery RT, GE Healthcare, Chicago, IL, USA). The target volumes and organs at risk (OARs) were delineated by the same clinician. The gross tumor volume (GTV) consisted of GTV of the primary (GTVp) and GTV of lymph nodes (GTVn). The clinical target volume (CTV) consisted of CTVp and CTVn. The planned target volume (PTV) included PTVp, PTVn, and PTV. All the GTVs, CTVs, and PTVs were contoured by the same oncologist based on international guidelines [10].

\section{Treatment plans and uncertainty plans}

A 2-arc volumetric-modulated arc therapy (VMAT) plan was generated for each patient using Varian Eclipse (13.6 Version,) treatment planning system (TPS) modeled for the VitalBeam (Varian, Palo Alto, US) linac. Arc 1 (A1) rotate clockwise from $181^{\circ}$ to $179^{\circ}$, and the arc 2 (A2) rotates counterclockwise from $179^{\circ}$ to $181^{\circ}$. Collimator angles were set at $\pm 10^{\circ}$. The prescription doses of PTVp, PTVn, and PTV were 69.96 Gy, 68.31 Gy, and 59.40 Gy in 33 fractions, respectively.

5 set-up uncertainties were introduced on the original VMAT plan, shifting the isocenter from its reference position according to the set-up errors acquired by CBCT. The U-plans, representing the perturbed plans introduced set-up uncertainties, were calculated for 33

Table 1 Patients characteristics

\begin{tabular}{llll}
\hline Patient \# & Age & Sex & Stage \\
\hline 1 & 57 & Male & T1N1M0 \\
2 & 62 & Male & T4N2M0 \\
3 & 68 & Male & T3N2M0 \\
4 & 27 & Male & T1N2M0 \\
5 & 28 & Female & T1N1M0 \\
6 & 68 & Male & T1N1M0 \\
7 & 67 & Male & T2N2M0 \\
8 & 43 & Male & T3NOM0 \\
9 & 54 & Male & T1N1M0 \\
10 & 38 & Male & T1N1M0 \\
\hline
\end{tabular}


Table 2 Evaluated items of PTVs and OARs

\begin{tabular}{|c|c|}
\hline PTVs/OARs & Evaluated items \\
\hline PTVp, PTVn, PTV & $\begin{array}{l}\Delta D_{2 c c^{\prime}} \Delta D_{98 \% \prime} \\
\Delta D_{95 \%,} \Delta D_{\text {mean }} \\
\Delta T C P\end{array}$ \\
\hline \multicolumn{2}{|l|}{ CTVp, CTVn, CTV } \\
\hline \multicolumn{2}{|l|}{ GTVp, GTVn } \\
\hline Brain Stem, Brain Stem PRV & $\triangle \mathrm{D}_{\max }, \triangle \mathrm{NTCP}$ \\
\hline \multicolumn{2}{|l|}{ Spinal Cord, Spinal Cord PRV } \\
\hline \multicolumn{2}{|l|}{ Lens L, Lens R } \\
\hline \multicolumn{2}{|l|}{ Optic Nerve L, Optic Nerve R, Optic Chiasma } \\
\hline Parotid L, Parotid R & $\triangle \mathrm{D}_{\text {mean, }}, \mathrm{NTCP}$ \\
\hline \multicolumn{2}{|c|}{$\begin{array}{l}\text { PTV, planning target volume; PTVp, planning target volume of GTVp; PTVn, } \\
\text { planning target volume of GTVn; CTV, clinical target volume; CTVp, primary } \\
\text { tumor sites and their invasion range; CTVn, the clinical target volume of GTVn; } \\
\text { GTVp, the clinical target volume of GTVp; GTVn, cervical metastatic lymph } \\
\text { node. } D_{\text {mean }} \text { and } D_{\text {max }} \text { represented the mean dose and the maximum dose. } D_{x \%} \\
\text { represented the dose (in Gy) received by } x \% \text { of the volume, } V_{y} \text { Gy the volume (in } \\
\text { percentage) received by y Gy. } D_{2 c c} \text { the dose (in Gy) received by a volume of } 2 \\
\mathrm{~cm}^{3}\end{array}$} \\
\hline
\end{tabular}

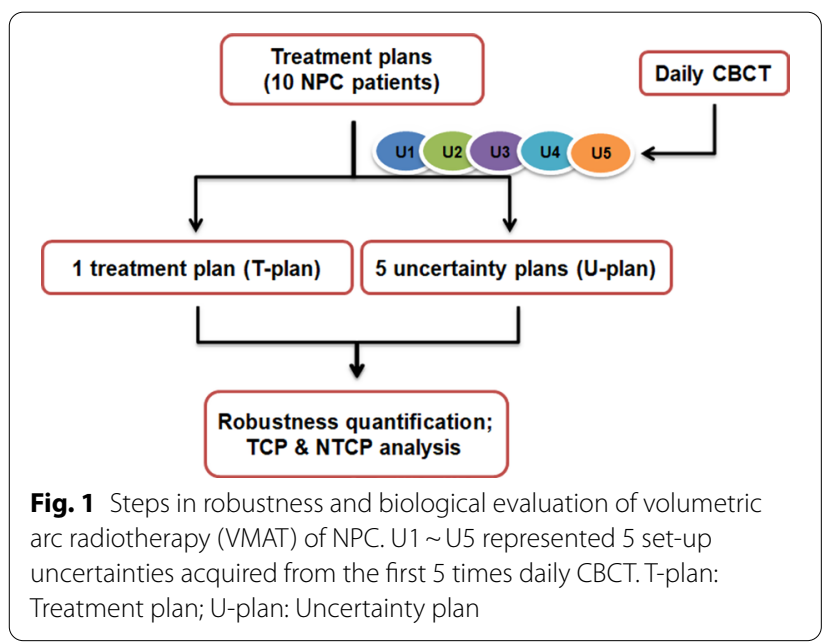

fractions to facilitate the dose comparison. The evaluated items of PTVs and OARs were listed in Table 2 (Fig. 1).

\section{Robustness quantification method}

There are 1 treatment plan (T-plan) and 5 uncertainty plans (U-plans) for each patient. The dose values in the treatment and perturbed plans were displayed in the dose-volume histogram (DVH) curves. $\mathrm{D}_{\mathrm{x} \%}$ represented the dose (in Gy) received by $x \%$ of the volume. $D_{2 c c}$ the dose (in Gy) received by a volume of $2 \mathrm{~cm}^{3}$. $D_{\max }$ and $\mathrm{D}_{\text {mean }}$ represented the maximum and mean dose (in Gy). Absolute differences $\Delta \mathrm{D}$, which could be calculated by the absolute value of the minimum value subtracted from the maximum value and corresponded to the plan robustness for the structure.

\section{TCP and NTCP evaluation}

Biological models have been proposed to predict radiobiological response to dose after irradiation $[11,12]$. The TCP and NTCP values were calculated to evaluate the biological effects. We use the Schultheiss logit model proposed by Niemierko [13]. We calculated the TCP according to Eq. (1) with the parameters: $\mathrm{TCD}_{50}=61.59 \mathrm{~Gy}$, $\gamma_{50}=3.38[14]$.

$$
T C P=\frac{1}{1+\left(\frac{T C D_{50}}{E U D}\right)^{4 \gamma_{50}}}
$$

$\mathrm{TCD}_{50}$ is the dose of radiation that locally controls $50 \%$ of tumors. The $\gamma_{50}$ is the change in TCP expected because of a $1 \%$ change in dose about the $\mathrm{TCD}_{50}$. We calculated the NTCP [14] according to Eq. (2)

$$
N T C P=\frac{1}{\sigma \sqrt{2 \pi}} \int_{-\infty}^{E U D} e^{-\left(\frac{\left(\mathrm{x}-T D_{50}\right)^{2}}{2 \sigma^{2}}\right)} d x
$$

The $\sigma$ was calculated by Eq. (3)

$$
\sigma=m T D_{50}
$$

The EUD, representing equivalent uniform dose, was calculated according to Eq. (4)

$$
E U D=\left(\frac{\sum_{i} V_{i} D_{i}^{1 / n}}{\sum_{i} V_{i}}\right)^{n}
$$

$\mathrm{TD}_{50}$ is the tolerance dose yielding a $50 \%$ complication rate in the normal organ. $\mathrm{V}_{\mathrm{i}}$ is the volume at dose $\mathrm{D}_{\mathrm{i}}$. Parameter $\mathrm{m}$ and $\mathrm{n}$ are specific dose-response constants [15].

\section{Statistical analysis}

There are 1 T-plan and $5 \mathrm{U}$-plans for each patient. The dose differences were calculated by the absolute value of the minimum value subtracted from the maximum value and were explicit by mean value (minimum value to maximum value). The dose deviations of $\mathrm{D}_{95 \%}, \mathrm{D}_{98 \%}, \mathrm{D}_{2 \mathrm{cc}}$, and $\mathrm{D}_{\text {mean }}$ of CTVs, GTVs, and PTVs were chosen. $\mathrm{D}_{\max }$ was chosen for serial OARs and $D_{\text {mean }}$ for the bilateral parotid gland. The TCP and NTCP reduction were calculated.

\section{Results \\ Targets dose coverage}

Figure 2 shows a schematic of transversal dose distributions in 1 treatment plan and $5 \mathrm{U}$-plans. The transversal dose coverage varies due to set-up uncertainty. To 
visualize the dose difference, a color wash schematic of differences in dose distributions is shown in Fig. 3. The maximum dose discrepancies were observed in marginal zones of PTVs. The dose changes of OARs were also greater in the vicinity of marginal zones and lesser distal to these areas.

The average dose difference was shown in Table 3. No obvious differences were found in $\mathrm{D}_{2 \text { cc. }}$. The mean dose differences of $D_{98 \%}$ and $D_{95 \%}$ of PTVp were respectively
3.30 Gy and 2.02 Gy. Decreased $\Delta \mathrm{D}_{98 \%}(1.12 \mathrm{~Gy})$ and $\Delta \mathrm{D}_{95 \%}(0.58 \mathrm{~Gy})$ were seen in CTVp. The $\Delta \mathrm{D}_{98 \%}$ and $\Delta \mathrm{D}_{95 \%}$ in GTVp were $0.56 \mathrm{~Gy}$ and $0.33 \mathrm{~Gy}$, indicating that the CTV-to-PTV margin promoted the robustness of GTV and CTV. Similarly, the PTVn had the largest difference of $\mathrm{D}_{98 \%}(2.77 \mathrm{~Gy})$ and $\mathrm{D}_{95 \%}(2.00 \mathrm{~Gy})$. The $\Delta \mathrm{D}_{98 \%}$ and $\Delta \mathrm{D}_{95 \%}$ of CTVn were 1.39 Gy and1.03 Gy. Minor dose differences were observed in GTVn for both $\mathrm{D}_{98 \%}(0.64 \mathrm{~Gy})$ and $\mathrm{D}_{95 \%}(0.59 \mathrm{~Gy})$. No marked mean dose variations of

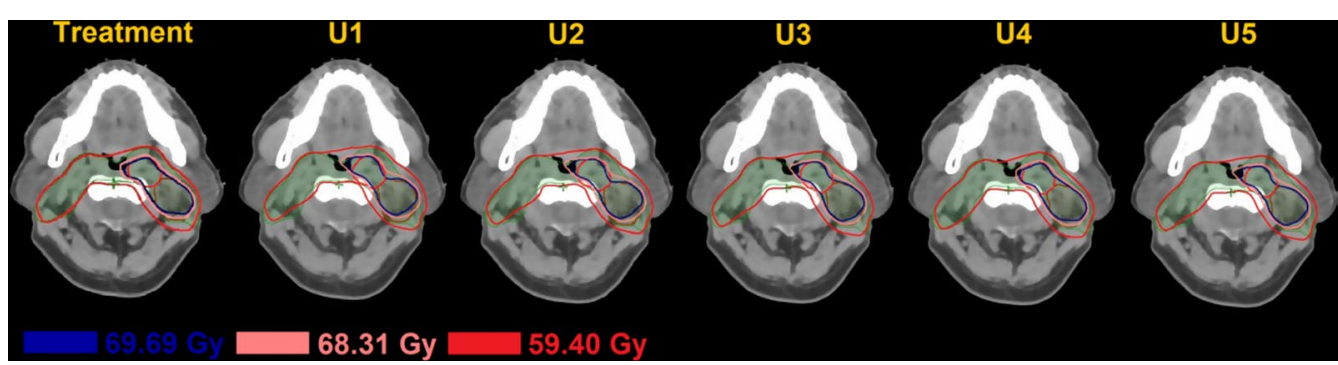

Fig. 2 A schematic of transversal dose distributions in 1 treatment plan and $5 \mathrm{U}$-plans. The green volume represents PTV, the brown volume represents PTVn, and the red volume represents PTVp. The transversal dose coverage varies due to set-up uncertainty

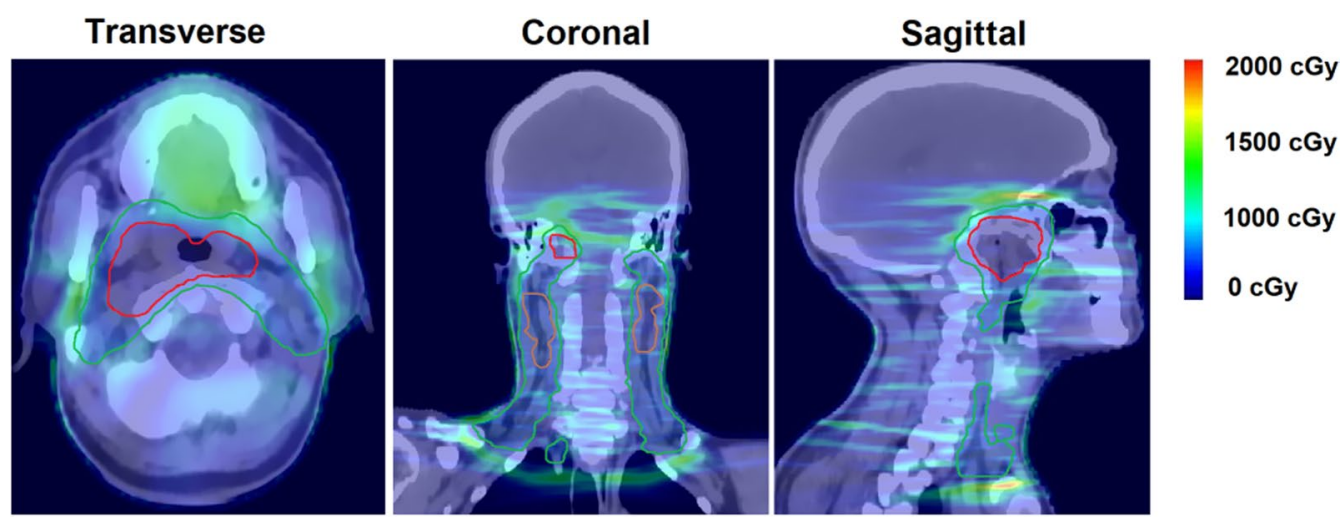

Fig. 3 An example of color wash schematic of dose difference bewteen the T-plan and one of the U-plan. The green, brown, and red volumes represent PTV, PTVn, and PTVp respectively

Table 3 Dose difference of PTVs, CTVs, and GTVs in 10 patients

\begin{tabular}{lllll}
\hline Targets & $\boldsymbol{\Delta D}_{\mathbf{2 c c}}(\mathrm{Gy})$ & $\boldsymbol{\Delta D}_{\mathbf{9 8 \%}}(\mathrm{Gy})$ & $\boldsymbol{\Delta D}_{\mathbf{9 5 \%}}(\mathrm{Gy})$ & $\boldsymbol{\Delta}$ \\
\hline PTVp & $0.23(0.07-0.34)$ & $3.30(0.76-4.38)$ & $2.02(0.53-3.23)$ & $0.31(0.11-0.70)$ \\
PTVn & $0.36(0.05-0.86)$ & $2.77(0.60-6.05)$ & $2.00(0.72-4.56)$ & $0.65(0.10-1.52)$ \\
PTV & $0.18(0.03-0.35)$ & $1.95(0.26-3.20)$ & $1.34(0.27-2.01)$ & $0.35(0.05-0.57)$ \\
CTVp & $0.20(0.04-0.35)$ & $1.12(0.38-3.85)$ & $0.58(0.23-1.50)$ & $0.16(0.05-0.30)$ \\
CTVn & $0.40(0.08-0.75)$ & $1.39(0.17-5.81)$ & $1.03(0.43-4.10)$ & $0.56(0.12-1.43)$ \\
CTV & $0.19(0.03-0.34)$ & $1.17(0.12-2.44)$ & $0.72(0.11-1.51)$ & $0.28(0.06-0.46)$ \\
GTVp & $0.28(0.12-0.37)$ & $0.56(0.06-2.88)$ & $0.33(0.07-0.67)$ & $0.22(0.03-0.49)$ \\
GTVn & $0.39(00.16-0.74)$ & $0.64(0.30-1.91)$ & $0.59(0.16-1.59)$ & $0.44(0.08-0.84)$ \\
\hline
\end{tabular}

The results were exhibited by Mean (Minimum-Maximum) 
Table 4 Dose difference of $D_{\text {max }}$ or $D_{\text {mean }}$ in OARs in 10 patients

\begin{tabular}{lll}
\hline Items & OARs & Dose (Gy) \\
\hline$\Delta \mathrm{D}_{\max }$ & Brain Stem & $4.34(1.50-11.10)$ \\
& Brain Stem PRV & $6.21(2.40-10.19)$ \\
& Spinal Cord & $2.86(1.00-7.10)$ \\
& Spinal Cord PRV & $3.54(1.70-7.40)$ \\
& Lens L & $0.89(0.20-2.80)$ \\
& Lens R & $0.79(0.20-1.40)$ \\
& Optical Nerve L & $8.00(1.80-17.40)$ \\
& Optical Nerve R & $8.66(1.40-20.20)$ \\
& Optic Chiasma & $8.81(1.80-20.80)$ \\
$\Delta D_{\text {mean }}$ & Parotid L & $4.48(2.47-7.28)$ \\
& Parotid R & $4.05(2.01-6.00)$ \\
\hline
\end{tabular}

The results were exhibited by Mean (Minimum-Maximum)
$\mathrm{D}_{\text {mean }}$ were seen. Superior robustness in PTV and CTV was seen.

Table 4 showed the dose differences of OARs. The $\Delta \mathrm{D}_{\max }$ of the brain stem and PRV were 4.34 Gy (1.50 Gy-11.10 Gy) and 6.21 Gy (2.40 Gy-10.19 Gy). The $\Delta D_{\max }$ of the spinal cord and PRV were 2.86 Gy (1.00 Gy-7.10 Gy) and 3.64 Gy (1.70 Gy-7.40 Gy). Narrowed width of DVH bands was observed in the bilateral lens. Optical nerves performed marked dose difference of mean dose, which were $8.00 \mathrm{~Gy}, 8.66 \mathrm{~Gy}$, and 8.81 Gy for optical nerve $\mathrm{L}, \mathrm{R}$, and chiasma. The $\mathrm{D}_{\text {mean }}$ of bilateral parotid glands exhibited obvious changes.

A sample of dose-volume histograms (DVHs) of PTVs, CTVs, and GTVs was shown in Fig. 4. The solid line represented the DVH of the treatment plan, and the 5 dashed lines represented the DVH of U-plans. The envelope was defined as the area between all the DVH
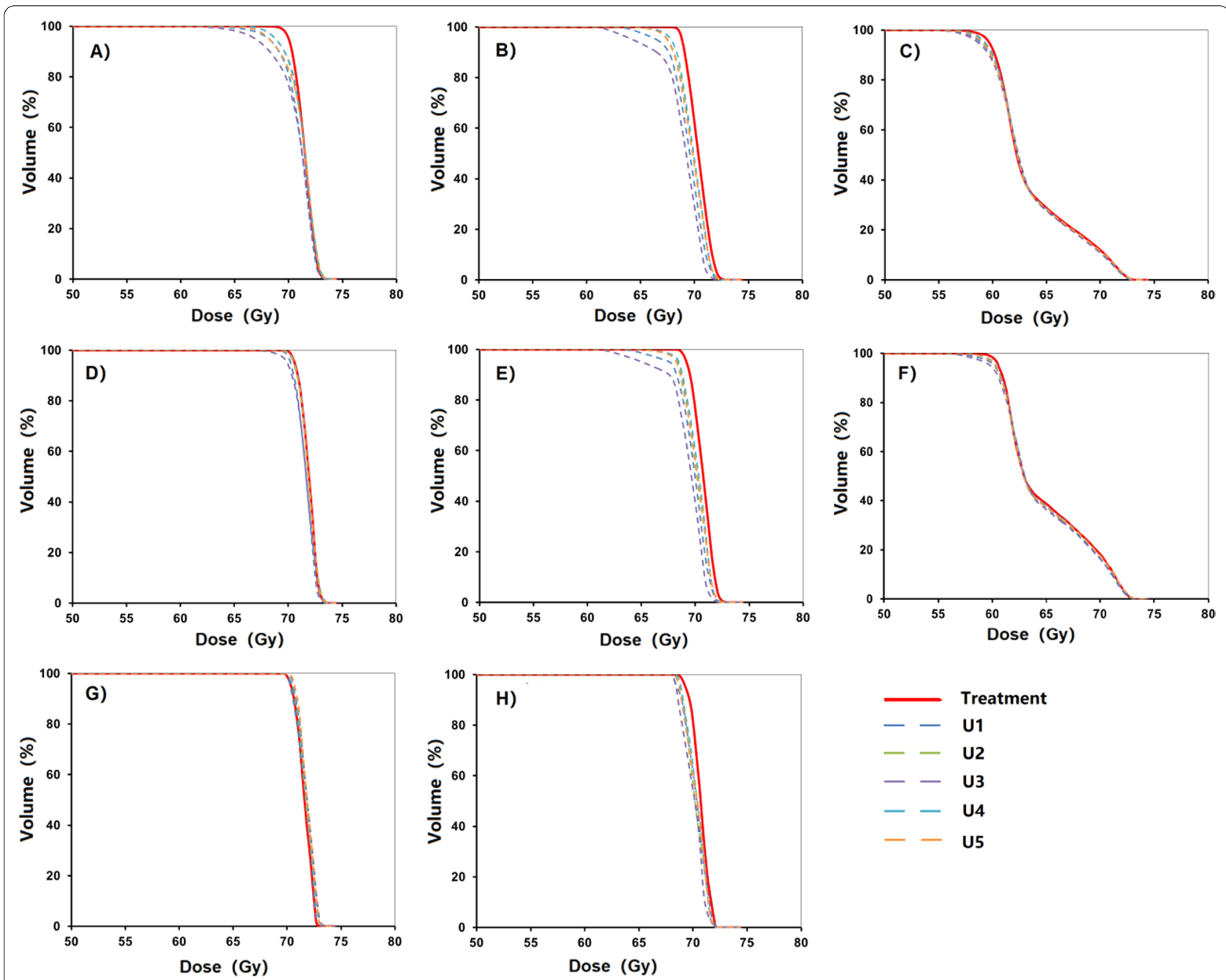

Fig. 4 A sample of dose-volume histograms (DVHs) of PTVs, CTVs, and GTVs, compared for 1 treatment plan and 5 U-plans. The DVH curve in the solid line represented the treatment plan. The DVH curves in the dashed line represented 5 U-plans. A PTVp; B PTVn; C PTV; D CTVp; E CTVn; F CTV; G GTVn; H GTVp 
curves. The gradually narrowed envelope was seen in PTVp (Fig. 4A), CTVp (Fig. 4D), and GTVp (Fig. 4G). PTVn (Fig. 4B) exhibited high sensitivity to set-up uncertainty. Narrowed width of the envelope was seen in CTVn (Fig. 4E). Sufficient dose coverage and decreased robustness were noticed in GTVn (Fig. 4H).
Superior robustness was seen in PTV (Fig. 4C) and CTV (Fig. 4F).

As to OARs (Fig. 5), the brain stem (Fig. 5A) and its PRV (Fig. 5B) exhibited weak robustness due to their locations in the vicinity of PTVs. The spinal cord (Fig. 5C) and its PRV (Fig. 5D) had stronger robustness.
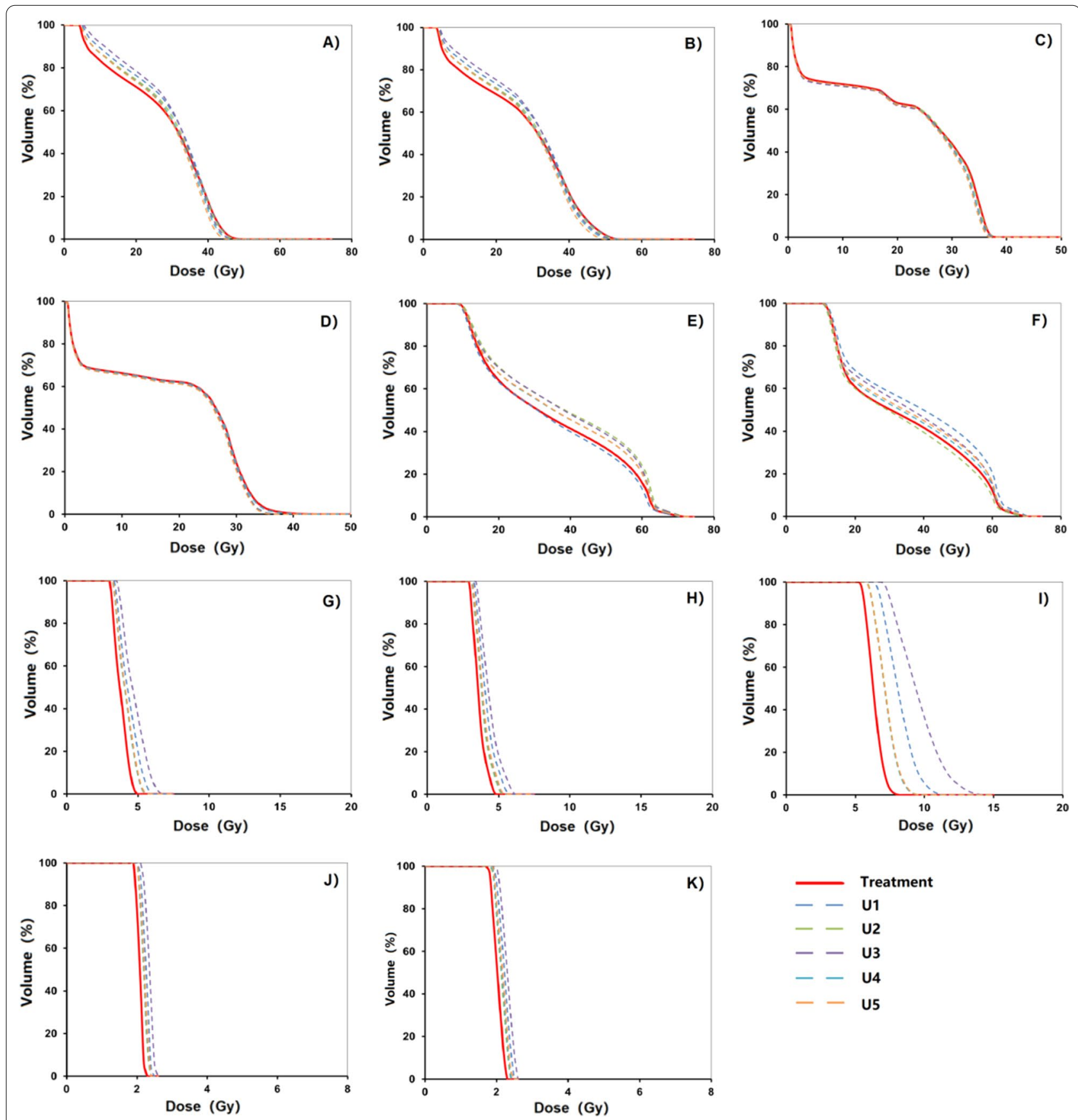

Fig. 5 A sample of dose-volume histograms (DVHs) of OARs was compared for 1 treatment plan and 5 U-plans. The DVH curve in the solid line represented the treatment plan. The DVH curves in the dashed line represented 5 U-plans. A Brain Stem; B Brain Stem PRV; C Spinal Cord; D Spinal Cord PRV; E Parotid L; F Parotid R; G Optical Nerve L; H Optical Nerve R; I Optical Nerve Chimsa; J Lens L; K Lens R 
Bilateral parotid glands (Fig. 5E. F) were sensitive to set-up uncertainty for their being partially enclosed PTVs. The $\mathrm{D}_{\max }$ of bilateral optical nerves (Fig. 5G-I) and lens(Fig. 5J, K) varied slightly.

\section{TCP and NTCP evaluation}

The TCP reduction $(\triangle \mathrm{TCP})$ was the mean absolute value of the minimum value subtracted from the maximum value. For GTVp and CTVp, the $\triangle \mathrm{TCP}$ value was less than $1 \%$ (Fig. 6), indicating strong robustness to set-up uncertainty. A greater $\triangle T C P$ value was observed in GTVn and CTVn. CTV had the largest TCP reduction.

We performed NTCP modeling analysis to evaluate the dose variation of OARs (Fig. 7). The NTCP reduction $(\triangle \mathrm{NTCP})$ was obtained as the mean absolute value of the minimum value subtracted from the maximum value. The average $\triangle \mathrm{NTCP}$ of bilateral parotids reached $6.17 \%$ (left) and 7.70\% (right) (Fig. 7). No significant biological dose changes were found in OARs.

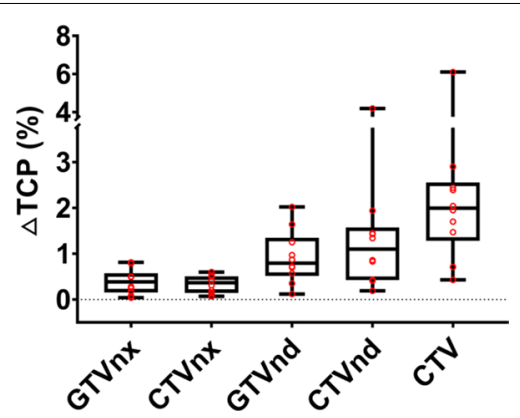

Fig. 6 Box plot showed the $\triangle T C P$ of all targets due to set-up uncertainties. The $\triangle T C P$ was the mean reduction of TCP

\section{Discussion}

VMAT plans exhibited strong sensitivity to geometric deviation PTVp and PTVn with large $\Delta D_{98 \%}$ and $\Delta \mathrm{D}_{95 \%}$. In photon radiotherapy, the CTV-to-PTV margin method was adopted based on the Van Herk margin formula [16] in the margin-based treatment planning, to ensure the dose coverage of CTV by blurring dose distribution induced by systematic setup errors. Although the CTV-to-PTV margin increased robustness in CTVp and CTVn, the $\Delta \mathrm{D}_{98 \%}$ of CTVp and CTVn reached 1.12 Gy and 1.39 Gy. The $\Delta D_{98 \%}$ of GTVp and GTVn reached 0.56 Gy and 0.64 Gy. Similarly, considerable dose deviations were observed in $\mathrm{D}_{95 \%}$ of CTVp, CTVn, PTVp, and PTVn. Although the margin method effectively improved the plan's robustness by reducing sensitivity to the uncertainties, high risk remains. The dose variation of $\mathrm{D}_{95 \%}$ and $\mathrm{D}_{98 \%}$ in PTVs could reach a maximum of $6 \mathrm{~Gy}$. The maximum difference of $\mathrm{D}_{95 \%}$ and $\mathrm{D}_{98 \%}$ in CTVs and GTVs could reach a maximum of $2.81 \mathrm{~Gy}$. The maximum difference of $D_{\text {mean }}$ of PTVs could reach $1.5 \mathrm{~Gy}$. The study of Dupic [17] indicated that the GTV $D_{98 \%}$ is a strong reproducible significant predictive factor of local control for the brain. A sufficient dose of GTVs should be rigidly reached. Zhao et al. [18] performed a retrospective study of a total of 1,092 patients with NSCLC of clinical-stage T1-T2 N0M0 who were treated with SABR. They recommended that both PTV $\mathrm{D}_{95 \%}$ and PTV $\mathrm{Pean}_{\text {mould be }}$ considered for plan optimization other than gross tumor volume. When the physical dose changed, the biological effect followed. The $\triangle \mathrm{TCP}$ in GTVp and CTVp were respectively $0.4 \%$ and $0.3 \%$. However, $\triangle \mathrm{TCP}$ of GTVn and CTVn were $0.92 \%$ and $1.3 \%$ respectively. The CTV had the largest mean variation of $\triangle \mathrm{TCP}(2.2 \%)$. Under dosage in the targets may result in the likelihood of tumor recurrence [19], for TCP predominately correlates
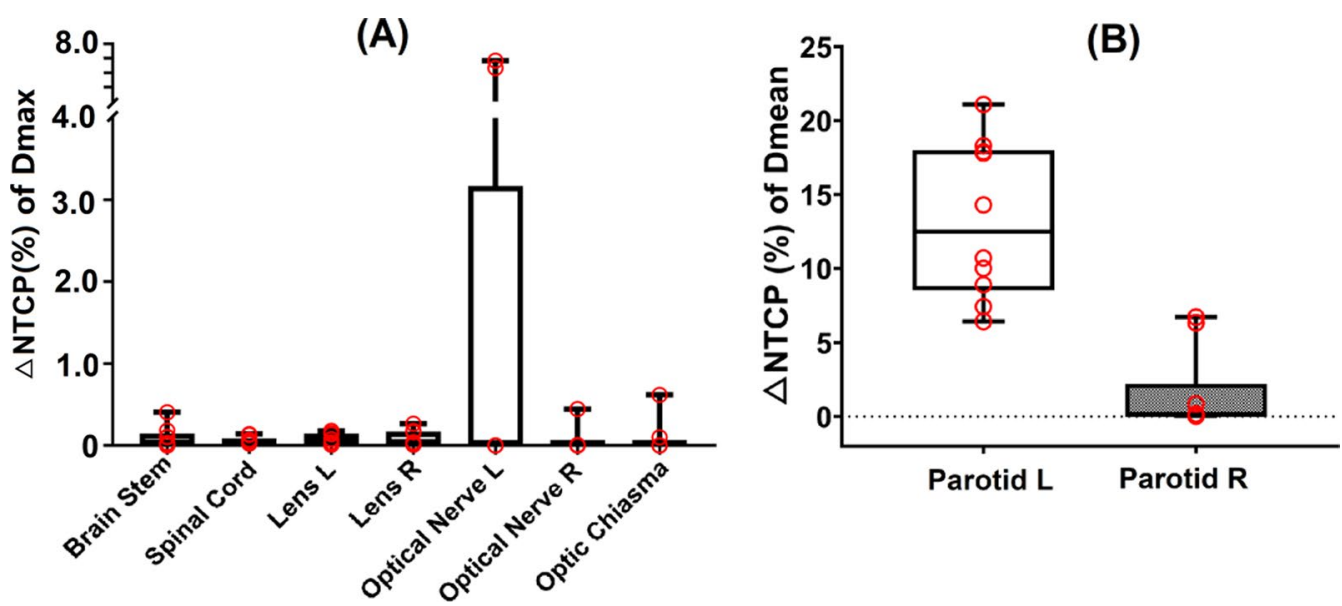

Fig. 7 Box plot showed the $\triangle N T C P$ of OARs due to set-up uncertainties. The $\triangle N T C P$ was the mean reduction of NTCP 
with the minimum dose of tumor [13]. Plan robustness of photon radiotherapy should be taken into consideration.

Weak robustnesses and large dose variations were observed in the OARs in the vicinity locations of PTVs. In this study, the average $\Delta D_{\max }$ of the brain stem and spinal cord reached 1.85 Gy and 1.51 Gy. Previous research reported that brain stem necrosis, MIR-based evidence of injury, or neurologic toxicities were related to photon radiotherapy [20-22]. Using conventional fractionation of 1.8-2 Gy/fraction to the full-thickness cord, the estimated risk of myelopathy is $<1 \%$ and $<10 \%$ at 54 Gy and 61 Gy, respectively [23]. For bilateral optic nerves and chiasm, the average $\Delta \mathrm{D}_{\max }$ were $4.59 \mathrm{~Gy}, 5.00 \mathrm{~Gy}$ and 5.01 Gy. There is a shred of strong evidence that evidence radiation tolerance is increased with a reduction in the dose per fraction [14, 24]. In radiotherapy of NPC, the bilateral parotids are often under irradiation. Salivary dysfunction has been correlated to the mean parotid gland dose, with recovery occurring with time [25-27]. The average $\triangle \mathrm{NTCP}$ of bilateral parotids reached $6.17 \%$ (left) and 7.70\% (right), which sharply increased the risk of parotid gland dysfunction. The actual irradiation dose of vicinal OAR may be biased upwards due to the set-up uncertainty.

Based on the results in this study, it is not hard to notice the strong sensitivity of highly optimized VMAT plans to geometric deviations. This generates worries about the accuracy of treatment dose delivery. 'Plan quality assessment' had been proposed firstly by the 3rd Physics ESTRO Workshop in 2019. Plan quality could be understood as the clinical suitability of the delivered dose distribution that can be realistically expected from a treatment plan [4]. Plan quality depends on the plan robustness and complexity of the treatment plan.

Intricate anatomical structures, precise dose coverage, and optimal OARs sparing generated highly optimized VMAT plans in NPC radiotherapy. High-degree modulated radiotherapy techniques increased plan complexity, with modulation of machine parameters, such as gantry rotate speed, continuously varied dose rate, and position of MLC. A study by Hirashima [28] uses plan complexity and dosiomics features to predict the performance for gamma passing rate, indicating the correlation between plan complexity and the accuracy of treatment plan dose delivery. Many commercial TPSs now offer the possibility to control plan complexity, such as controlling the minimum size and monitor unit (MU) (Phillips Pinnacle, Amsterdam, the Netherlands), aperture shape controller (ASC) (Varian Eclipse, Palo Alto, CA, USA), and modulation factor (MF) (TomoTherapy, Accuray Incorporated, Sunnyvale, CA, USA). The balance should be reached between dosimetric improvement and dose delivery accuracy.
Plan robustness qualification was always considered in proton therapy to address sensitivity to uncertainties in treatment planning [29]. In photon RT, the CTV-to-PTV margin method had been adopted to assure dose coverage with uniform margin, instead of plan robustness qualification. However, the CTV-to-PTV margin method has limitations, such as relying on the so-called static dose cloud approximation. A phantom study conducted by Englesman et al. [30] observed a maximum decreased dose of $5 \%$ with respiratory motion uncertainty. Guerreiro [31] evaluated the robustness against inter-fraction anatomical changes between photon and proton dose distributions and found that daily anatomical changes proved to affect the target coverage of VMAT dose distributions to a higher extent. Our results indicated that CTV-to-PTV margin increased robustness of CTV and GTV, reduced but did not remove the risk of underdosage. This plan robustness quantification method could be adopted in highly optimized clinical treatment plans to make a more complete dose description.

Besides, the robustness optimization methods had been developed by incorporating uncertainty in plan optimization, for CTV should receive the prescribed dose depending on desired dose distribution and dose fall-off near the target rather than geometric margin [32]. Lowe et al. [33] believed robustness optimization was an effective method to reduce dose to normal tissues that would be unnecessarily irradiated with the CTV-to-PTV margin concept. Dosimetric consequences of uncertainty, such as equivalent uniform dose (EUD), TCP, and NTCP were also recommended.

Among the limitation of the study, it is important to highlight that the first 5 times set-up errors acquired from $\mathrm{CBCT}$ did not represent the actual set-up uncertainty, for the set-up error consisted of systematic and random errors. Additionally, the patient anatomy change and rotation have not been taken into account. As a possible solution, adaptive radiotherapy (ART) could help to solve this problem [34]. We aimed to simulate the scenarios introduced to set up uncertainties, and visualize the necessity of robustness quantification is highly optimized photon RT. Treatment plan robustness analysis provides a more complete description of the dose delivered in the presence of uncertainties, and may lead to future dosimetric studies with improved accuracy.

\section{Conclusions}

VMAT plans had a strong sensitivity to set-up uncertainty in NPC radiotherapy, due to the high degree of modulation. We proposed an effective method to evaluate the plan robustness of VMAT plans. Plan robustness and complexity should be taken into account in photon radiotherapy techniques with high degree optimization. 
The robust optimization may have the potential and could be considered in complex plans with a reliable evaluation of long-term clinical outcomes.

\begin{abstract}
Abbreviations
PTV: Planning target volume; PTVp: Planning target volume of GTVnx; PTVn: Planning target volume of GTVnd; CTV: Clinical target volume; CTVp: Primary tumor sites and their invasion range; CTVn: The clinical target volume of GTVn; GTVp: The clinical target volume of GTVp; GTVn: Cervical metastatic lymph node; CBCT: Cone beam computed tomography; VMAT:Volumetric arc radiotherapy; IMRT: Intentively modulated radiotherapy; 3D-CRT: 3-Dimension conformal radiotherapy; OAR: Organs at risk; TCP: Tumor control probability; NTCP: Normal tissues complication probability.
\end{abstract}

\section{Acknowledgements}

Not applicable.

\section{Authors' contributions}

ZD: Methodology, Data Curation; Writing; XX: Conceptualization, Methodology; QZ, JM: Data Curation and preparation of tables; ZD, KK, SB: Preparation of figures. All authors read and approved the final manuscript.

\section{Funding}

The research was supported by Sanming Project of Medicine in Shenzhen (SZSM201612063), Shenzhen Key Medical Discipline Construction Fund (SZXK013), and Shenzhen High-level Hospital Construction Fund.

\section{Availability of data and materials}

The datasets used and/or analyzed during the current study are available from the corresponding author on reasonable request.

\section{Declarations}

\section{Ethics approval and consent to participate}

The study was approved by the institutional review board of National Cancer Center/National Clinical Research Center for Cancer/Cancer Hospital \& Shenzhen Hospital. We confirm that all methods were carried out in accordance with relevant guidelines and regulations.

\section{Consent for publication}

The patients gave their informed consent for use of the data for research purposes.

\section{Competing interests}

The authors state that they have no competing interests.

Received: 30 August 2021 Accepted: 17 December 2021

Published online: 03 January 2022

\section{References}

1. Sun XS, Li XY, Chen QY, Tang LQ, Mai HQ. Future of radiotherapy in nasopharyngeal carcinoma. Br J Radiol. 2019;92(1102):20190209. https://doi. org/10.1259/bjr.20190209.

2. Lee HM, Okuda KS, González FE, Patel V. Current perspectives on nasopharyngeal carcinoma. Adv Exp Med Biol. 2019;1164:11-34. https://doi. org/10.1007/978-3-030-22254-3_2.

3. Huang TL, Tsai MH, Chuang HC, et al. Quality of life and survival outcome for patients with nasopharyngeal carcinoma treated by volumetricmodulated arc therapy versus intensity-modulated radiotherapy. Radiat Oncol. 2020;15(1):84. https://doi.org/10.1186/s13014-020-01532-4.

4. Hernandez $V$, Hansen $C R$, Widesott $L$, et al. What is plan quality in radiotherapy? The importance of evaluating dose metrics, complexity, and robustness of treatment plans. Radiother Oncol. 2020;153:26-33. https:// doi.org/10.1016/j.radonc.2020.09.038.
5. Korevaar EW, Habraken SJM, Scandurra D, et al. Practical robustness evaluation in radiotherapy - a photon and proton-proof alternative to PTV-based plan evaluation. Radiother Oncol. 2019;141:267-74. https:// doi.org/10.1016/j.radonc.2019.08.005.

6. Hubley E, Pierce G. The influence of plan modulation on the interplay effect in VMAT liver SBRT treatments. Phys Med. 2017;40:115-21. https:// doi.org/10.1016/j.ejmp.2017.07.025.

7. Boda-Heggemann J, Lohr F, Wenz F, Flentje M, Guckenberger M. kV cone-beam CT-based IGRT: a clinical review. Strahlenther Onkol. 2011;187(5):284-91. https://doi.org/10.1007/s00066-011-2236-4.

8. Wang $\mathrm{H}$, Huang $\mathrm{Y}, \mathrm{Hu} \mathrm{Q}$, et al. A simulated dosimetric study of contribution to radiotherapy accuracy by fractional image guidance protocol of halcyon system. Front Oncol. 2021;10:543147. https://doi.org/10.3389/ fonc.2020.543147.

9. Yock AD, Mohan R, Flampouri S, et al. Robustness analysis for external beam radiation therapy treatment plans: describing uncertainty scenarios and reporting their dosimetric consequences. Pract Radiat Oncol. 2019;9(4):200-7. https://doi.org/10.1016/j.prro.2018.12.002.

10. Lee AW, Ng WT, Pan JJ, et al. International guideline for the delineation of the clinical target volumes (CTV) for nasopharyngeal carcinoma. Radiother Oncol. 2018;126(1):25-36. https://doi.org/10.1016/j.radonc.2017.10. 032.

11. Liu F, Tai A, Lee P, et al. Tumor control probability modeling for stereotactic body radiation therapy of early-stage lung cancer using multiple bio-physical models. Radiother Oncol. 2017;122(2):286-94. https://doi. org/10.1016/j.radonc.2016.11.006.

12. Jakobi A, Lühr A, Stützer K, et al. Increase in tumor control and normal tissue complication probabilities in advanced head-and-neck cancer for dose-escalated intensity-modulated photon and proton therapy. Front Oncol. 2015;5:256. https://doi.org/10.3389/fonc.2015.00256.

13. Gay HA, Niemierko A. A free program for calculating EUD-based NTCP and TCP in external beam radiotherapy. Phys Med. 2007;23(3-4):115-25. https://doi.org/10.1016/j.ejmp.2007.07.001.

14. Mayo C, Martel MK, Marks LB, Flickinger J, Nam J, Kirkpatrick J. Radiation dose-volume effects of optic nerves and chiasm. Int J Radiat Oncol Biol Phys. 2010;76(3 Suppl):S28-35. https://doi.org/10.1016/j.jirobp.2009.07. 1753.

15. Luxton G, Keall PJ, King CR. A new formula for normal tissue complication probability (NTCP) as a function of equivalent uniform dose (EUD). Phys Med Biol. 2008;53(1):23-36. https://doi.org/10.1088/0031-9155/53/1/002.

16. Van Herk M. Errors and margins in radiotherapy. Semin Radiat Oncol. 2004;14(1):52-64. https://doi.org/10.1053/j.semradonc.2003.10.003.

17. Dupic G, Brun L, Molnar I, et al. Significant correlation between gross tumor volume (GTV) D98\% and local control in multifraction stereotactic radiotherapy (MF-SRT) for unresected brain metastases. Radiother Oncol. 2021;154:260-8. https://doi.org/10.1016/j.radonc.2020.11.021.

18. Zhao L, Zhou S, Balter P, et al. Planning target volume D95 and mean dose should be considered for optimal local control for stereotactic ablative radiation therapy. Int J Radiat Oncol Biol Phys. 2016;95(4):1226-35. https://doi.org/10.1016/j.ijrobp.2016.01.065.

19. Lu JY, Lin Z, Zheng J, Lin PX, Cheung ML, Huang BT. Dosimetric evaluation of a simple planning method for improving intensity-modulated radiotherapy for stage III lung cancer. Sci Rep. 2016;6:23543. https://doi.org/10. 1038/srep23543.

20. Uy NW, Woo SY, Teh BS, et al. Intensity-modulated radiation therapy (IMRT) for meningioma. Int J Radiat Oncol Biol Phys. 2002;53(5):1265-70. https://doi.org/10.1016/s0360-3016(02)02823-7.

21. Wenkel E, Thornton AF, Finkelstein D, et al. Benign meningioma: partially resected, biopsied, and recurrent intracranial tumors treated with combined proton and photon radiotherapy. Int J Radiat Oncol Biol Phys. 2000;48(5):1363-70. https://doi.org/10.1016/s0360-3016(00)01411-5.

22. Mayo C, Yorke E, Merchant TE. Radiation associated brainstem injury. Int J Radiat Oncol Biol Phys. 2010;76(3 Suppl):S36-41. https://doi.org/10. 1016/j.jijrobp.2009.08.078.

23. Kirkpatrick JP, van der Kogel AJ, Schultheiss TE. Radiation dose-volume effects in the spinal cord. Int J Radiat Oncol Biol Phys. 2010;76(3 Suppl):S42-9. https://doi.org/10.1016/j.ijrobp.2009.04.095.

24. Danesh-Meyer HV. Radiation-induced optic neuropathy. J Clin Neurosci. 2008;15(2):95-100. https://doi.org/10.1016/j.jocn.2007.09.004.

25. Deasy JO, Moiseenko V, Marks L, Chao KS, Nam J, Eisbruch A. Radiotherapy dose-volume effects on salivary gland function. Int J Radiat Oncol 
Biol Phys. 2010;76(3 Suppl):S58-63. https://doi.org/10.1016/j.jprobp.2009. 06.090.

26. Chao KS, Deasy JO, Markman J, et al. A prospective study of salivary function sparing in patients with head-and-neck cancers receiving intensitymodulated or three-dimensional radiation therapy: initial results. Int J Radiat Oncol Biol Phys. 2001;49(4):907-16. https://doi.org/10.1016/s03603016(00)01441-3.

27. Blanco Al, Chao KS, El Naqa I, et al. Dose-volume modeling of salivary function in patients with head-and-neck cancer receiving radiotherapy. Int J Radiat Oncol Biol Phys. 2005;62(4):1055-69. https://doi.org/10.1016/j. ijrobp.2004.12.076.

28. Hirashima $\mathrm{H}$, Ono T, Nakamura $\mathrm{M}$, et al. Improvement of prediction and classification performance for gamma passing rate by using plan complexity and dosiomics features. Radiother Oncol. 2020;153:250-7. https:// doi.org/10.1016/j.radonc.2020.07.031.

29. Unkelbach J, Alber M, Bangert M, et al. Robust radiotherapy planning. Phys Med Biol. 2018;63(22):22TR02. https://doi.org/10.1088/1361-6560/ aae659.

30. Engelsman M, Damen EM, De Jaeger K, van Ingen KM, Mijnheer BJ. The effect of breathing and set-up errors on the cumulative dose to a lung tumor. Radiother Oncol. 2001;60(1):95-105. https://doi.org/10.1016/ s0167-8140(01)00349-8.

31. Guerreiro F, Zachiu C, Seravalli E, et al. Evaluating the benefit of PBS vs. VMAT dose distributions in terms of dosimetric sparing and robustness against inter-fraction anatomical changes for pediatric abdominal tumors. Radiother Oncol. 2019;138:158-65. https://doi.org/10.1016/j. radonc.2019.06.025.

32. Dunlop A, Colgan R, Kirby A, Ranger A, Blasiak-Wal I. Evaluation of organ motion-based robust optimisation for VMAT planning for breast and internal mammary chain radiotherapy. Clin Transl Radiat Oncol. 2019;16:60-6. https://doi.org/10.1016/j.ctro.2019.04.004.

33. Lowe M, Aitkenhead A, Albertini F, Lomax AJ, MacKay RI. A robust optimisation approach accounting for the effect of fractionation on setup uncertainties. Phys Med Biol. 2017;62(20):8178-96. https://doi.org/10. 1088/1361-6560/aa8c58.

34. Castelli J, Simon A, Lafond C, et al. Adaptive radiotherapy for head and neck cancer. Acta Oncol. 2018;57(10):1284-92. https://doi.org/10.1080/ 0284186X.2018.1505053.

\section{Publisher's Note}

Springer Nature remains neutral with regard to jurisdictional claims in published maps and institutional affiliations.

Ready to submit your research? Choose BMC and benefit from:

- fast, convenient online submission

- thorough peer review by experienced researchers in your field

- rapid publication on acceptance

- support for research data, including large and complex data types

- gold Open Access which fosters wider collaboration and increased citations

- maximum visibility for your research: over 100M website views per year

At BMC, research is always in progress.

Learn more biomedcentral.com/submissions 